REPLY

\section{A further strategy to combat the high price of anticancer drugs}

\author{
Vinay Prasad, Kevin De Jesús and Sham Mailankody
}

We thank Mr Gilbar for his interest in our Perspectives article (The high price of anticancer drugs: origin, implications, barriers, solutions. Nat. Rev. Clin. Oncol. 14, 381-390 (2017) $)^{1}$, and his expansion on the approaches to tackling the problem of the high prices of anticancer drugs (A further strategy to combat the high price of anticancer drugs. Nat. Rev. Clin. Oncol. http:// dx.doi.org/10.1038.org/nrclinonc.2017.31-c1 $(2017))^{2}$. Mr Gilbar focuses on the issue of wastage that occurs when single-use vials of anticancer drugs are partially used, and the remaining drug discarded. As Mr Gilbar describes $^{2}$, ways to minimize such waste include the availability of smaller vials or a greater range of vial sizes, the use of multiuse containers, and others. These solutions are reasonable and must be considered by physicians and policy makers.

We would like to highlight two additional strategies for reducing the unnecessary expenditure on anticancer drugs that have been proposed recently. Firstly, in June 2017, Goldstein and colleagues ${ }^{3}$ reported that personalized dosing of pembrolizumab at $2 \mathrm{mg} /$ $\mathrm{kg}$ for patients with metastatic non-smallcell lung cancer would lead to annual savings of $\$ 825$ million in the USA, compared with the use of this agent at a fixed dose of $200 \mathrm{mg}$. Secondly, interest in conducting clinical trials focused on lowering the dose of anticancer drugs without compromising efficacy is increasing. For example, the Value in Cancer Care consortium (http://www.vi3c. org/), a nonprofit organization inaugurated in May 2017, reported results of a pilot clinical trial demonstrating that lower doses of abiraterone (up to three-quarters lower than the standard dose) taken with a low-fat breakfast has similar efficacy in men with prostate cancer to the standard dose taken on an empty stomach; the consortium are planning a larger follow-up study. Thus, we agree that improvements in the efficiency of anticancer drug dosing and administration can result in cost savings.

Nevertheless, focusing predominantly on wastage is unlikely to provide durable solutions to the problem of high drug prices. Previous work has shown that the costs of manufacturing anticancer drugs is low, and do not reflect the current prices of the drugs. For instance, Hill and colleagues ${ }^{5}$ have shown that the cost of formulation and packaging, including a $50 \%$ profit margin, for four FDA-approved tyrosine-kinase inhibitors (imatinib, erlotinib, lapatinib, and sorafenib) ranges between US $\$ 128$ to $\$ 4,020$ per personyear, whereas the annual prices of these drugs in the USA are between $\$ 75,161$ and $\$ 139,138$. Because drug prices are not linked to the manufacturing and packaging costs, manufacturers could adjust the prices to offset potential cost reductions associated with changes in vial size, personalized dosing, dose-rounding, and dose-banding. Take for instance ponatinib, a tyrosine-kinase inhibitor approved for the treatment of chronic myeloid leukaemia ${ }^{6}$ : the per-tablet price for the $15 \mathrm{mg}$ and $45 \mathrm{mg}$ formulations is identical at $\$ 534$, and the cost of a 30-day supply of $15 \mathrm{mg}$ or $45 \mathrm{mg}$ of ponatinib tablets would be approximately $\$ 16,024$ ('full cost of drug' as reported in the Plan Finder ${ }^{7}$ for Humana PDP Enhanced for a beneficiary living within ZIP code 10065). Owing to concerns regarding the toxicity of $45 \mathrm{mg}$ daily dosing, oncologists often treat patients with $30 \mathrm{mg}$ of ponatinib; however, $30 \mathrm{mg}$ tablets are not available, and the price of two $15 \mathrm{mg}$ tablets (for a total daily dose of $30 \mathrm{mg}$; \$1068) is twice that of a $45 \mathrm{mg}$ tablet. Although oncologists and policymakers in the USA have highlighted this issue ${ }^{8}$, the pricing of ponatinib has not been changed to date.

In summary, for the reasons outlined herein and in our Perspectives ${ }^{1}$, the list prices of anticancer drugs are prohibitively high and need to be addressed. Focusing on reducing wastage reinforces the notion that each drop of an anticancer drug has an immutable cost, and the high price of these drugs is related to the total dose administered; as discussed, this is not always the case. Thus, the strategies to optimize anticancer drug usage suggested by Mr Gilbar ${ }^{2}$ and others are complementary to lowering drug prices, but are, in our opinion, insufficient to address the fundamental issues with the high costs of anticancer drugs.

Vinay Prasad is at the Division of Hematology and Medical Oncology, Knight Cancer Institute, Department of Preventive Medicine and Public Health, and Center for Health Care Ethics, Oregon Health and

Science University, Portland, Oregon 97239, USA.

Kevin De Jesús is at Ponce Health Sciences UniversitySchool of Medicine, P.O. BOX 7004, Ponce, Puerto Rico 00716-2347, USA.

Sham Mailankody is at the Myeloma Service, Department of Medicine, Memorial Sloan Kettering Cancer Center, SR-505N3, Scholars Building, 5th Floor, 1233 York Avenue, New York, New York 10065, USA.

Correspondence to S.M. mailanks@mskcc.org doi:10.1038/nrclinonc. 2017.137 Published online 22 Aug 2017

1. Prasad, V., De Jesus, K. \& Mailankody, S. The high price of anticancer drugs: origin, implications, barriers, solutions. Nat. Rev. Clin. Oncol. 14, 381-390 (2017) 2. Gilbar, P. J. A further strategy to combat the high price of anticancer drugs. Nat. Rev. Clin. Oncol. http://dx. doi.org/10.1038.org/nrclinonc.2017.31-c1 (2017).

3. Goldstein, D. A. et al. A pharmacoeconomic analysis of personalized dosing versus fixed dosing of pembrolizumab in first-line PD-L1-positive non-small cell lung cancer. J. Clin. Oncol. 35 (Suppl.) abstr 9013 (2017).

4. McGinley, L. Cancer drug prices are so high that doctors will test cutting doses. www.washingtonpost. com https://www.washingtonpost.com/news/to-yourhealth/wp/2017/06/08/how-these-cancer-doctorsplan-to-reduce-patients-drug-costs-without-touchingprices/?utm term $=.616 \mathrm{~b} 7 \mathrm{f765faa}$ (2017).

5. Hill, A. et al. Target prices for mass production of tyrosine kinase inhibitors for global cancer treatment. BMJ Open 6, e009586 (2016).

6. Prasad, V. \& Mailankody, S. The accelerated approval of oncologic drugs: lessons from ponatinib. JAMA 311 353-354 (2014)

7. Center for Medicare and Medicaid Services. Medicare plan finder. www.medicare.gov https://www.medicare. gov/find-a-plan/questions/home.aspx (2017)

8. Hagen, $\mathrm{T}$. Ponatinib (CML) pricing gets a blast from congress. www.onclive.com http://www.onclive.com/ web-exclusives/ponatinib-cml-pricing-gets-a-blastfrom-congress (2016).

Competing interests statement

S.M. reports serving as a Principal Investigator of clinical trials with research funding from Juno Therapeutics and Takeda Oncology. V.P. and K.D.J. declare no competing interests.

FURTHER INFORMATION Value in Cancer Care consortium: http://www.vi3c.org/ ALL LINKS ARE ACTIVE IN THE ONLINE PDF 